\title{
An Efficient Algorithm for Unsupervised Word Segmentation with Branching Entropy and MDL
}

\author{
Valentin Zhikov*1 \\ Hiroya Takamura \\ Manabu Okumura
}

Interdisciplinary Graduate School of Science and Engineering, Tokyo Institute of Technology

zhikov@lr.pi.titech.ac.jp, http://www.lr.pi.titech.ac.jp/ ₹zikov/

Precision and Intelligence Laboratory, Tokyo Institute of Technology

takamura@pi.titech.ac.jp, http://www.lr.pi.titech.ac.jp/ takamura/

Precision and Intelligence Laboratory, Tokyo Institute of Technology

oku@pi.titech.ac.jp, http://www.Ir.pi.titech.ac.jp/ oku/

keywords: unsupervised word segmentation, semi-supervised word segmentation, branching entropy, minimum description length

\section{Summary}

This paper proposes a fast and simple unsupervised word segmentation algorithm that utilizes the local predictability of adjacent character sequences, while searching for a least-effort representation of the data. The model uses branching entropy as a means of constraining the hypothesis space, in order to efficiently obtain a solution that minimizes the length of a two-part MDL code. An evaluation with corpora in Japanese, Thai, English, and the "CHILDES" corpus for research in language development reveals that the algorithm achieves a F-score, comparable to that of the state-of-the-art methods in unsupervised word segmentation, in a significantly reduced computational time. In view of its capability to induce the vocabulary of large-scale corpora of domain-specific text, the method has potential to improve the coverage of morphological analyzers for languages without explicit word boundary markers. A semi-supervised word segmentation approach is also proposed, in which the word boundaries obtained through the unsupervised model are used as features for a state-of-the-art word segmentation method.

\section{Introduction}

As an inherent preliminary step to nearly all tasks that involve the automated processing of text in writing systems without orthographical marking of word boundaries, such as Japanese and Chinese, the importance of word segmentation has lead to the emergence of a microgenre in NLP focused exclusively on this problem.

Supervised probabilistic models such as conditional random fields [Lafferty 01] are presently employed for a highly accurate automated morphological analysis of these languages. However, the development of the annotated corpora necessary for their training is still a labor-intensive task, which involves multiple stages of manual curation. Because of the scarcity of labeled data, the domain adaptation of morphological analyzers is also problematic, and semi-supervised algorithms that address this issue have been proposed (e.g., [Liang 05, Tsuboi 08]). Apart from

$\dagger 1$ Currently, Valentin Zhikov works for Ontotext AD in Bulgaria. the considerable difficulties in discovering reliable criteria for word induction, the practical use of such approaches is impeded by their prohibitive computational cost.

In this paper, we address the issue of achieving a high word boundary prediction accuracy in a practical computational time through an efficient method that relies on a combination of evidences: the local predictability of character patterns, and the reduction of effort achieved by a given representation of the language data. Both of these criteria are assumed to play a key role in native language acquisition. The proposed model allows experimentation in a more realistic setting, where the learner is able to apply them simultaneously. The method shows a high performance in terms of F-score and speed, can be applied to language samples of substantial length, and generalizes well to corpora in different languages. A semi-supervised word segmentation approach is also proposed, in which the word boundaries obtained through the unsupervised model are used as features for developing a state-of-theart word segmentation model. 


\section{Related Work}

The principle of least effort [Zipf 49] postulates that the path of minimum resistance underlies all human behavior. Recent research has recognized its importance in the process of language acquisition [Kit 03]. Segmentation models based on compression rely on this principle, as they reorganize the data into a more compact representation, in order to induce the vocabulary of a text. The MDL framework [Rissanen 78] is an appealing means of formalizing such models, as it provides a robust foundation for learning and inference, based solely on compression.

The major issue with the MDL-based word segmentation is the lack of standardized search algorithms for the exponential hypothesis space [Goldwater 09]. The representative MDL models compare favorably to the current state-of-the-art models in terms of accuracy. Brent and Cartwright carried out an exhaustive search through the possible segmentations of a limited subset of the data [Brent 96]. Yu proposed an EM optimization routine [Yu 00], which achieved a high F-score, in spite of a lower compression rate than the one achieved by the referenced gold standard segmentation, thus confirming the strong relationship between the least-effort representation criterion and the capability of the model to correctly identify the lexicon of a document collection.

As a solution to the aforementioned issue, the proposed method incorporates the local predictability of character sequences into the inference process. Numerous studies have shown that local distributional cues can serve well the purpose of inducing word boundaries. Behavioral science research has confirmed that infants are sensitive to the transitional probabilities found in speech [Saffran 96]. The increase in uncertainty following a given word prefix is a well studied criterion for morpheme boundary prediction [Harris 55]. A good deal of research has been conducted on methods through which such local statistics can be applied to the word induction problem (e.g., [Kempe 99, Huang 03, Jin 06]). Hutchens and Adler [Hutchens 98] noticed that entropic chunking has the effect of reducing the perplexity of a text.

Most methods for the unsupervised word segmentation task based solely on local statistics presume a certain albeit minimum - level of acquaintance with the target language. For instance, the model of Huang and Powers [Huang 03] involves some parameters (Markov chain order, numerous threshold values) that allow its adaptation to the individuality of written Chinese. In comparison, the method proposed in this paper generalizes easily to a variety of languages and domains, and is less dependent on annotated development data.

The state-of-the-art in unsupervised word segmentation is represented by Bayesian models. Goldwater et al. [Goldwater 06] justified the importance of context as a means of avoiding undersegmentation, through a method based on hierarchical Dirichlet processes. Mochihashi et al. [Mochihashi 09] proposed extensions to this method, which included a nested character model and an optimized inference procedure. Johnson and Goldwater have proposed a novel method based on adaptor grammars, whose Fscore surpasses the aforementioned methods by a large margin, when appropriate assumptions are made regarding the structural units of a language [Johnson 09].

The technique that we propose in this paper unifies conclusions made by different strains of research (language acquisition and word segmentation), through which two independently studied information theoretic criteria (MDL, e.g., [Brent 96, Yu 00], the local predictability of character sequence, e.g., [Kempe 99, Huang 03, Jin 06]) are combined into a single efficient heuristic approach. Due to its practical nature, the proposed algorithm allows us to evaluate the effect of using huge datasets on the accuracy of unsupervised segmentation. One noticeable conclusion of this paper that is against the conclusions made in the past research is related to the effect of compression on accuracy; Yu showed that the reference segmentation is more efficient than the one discovered by their algorithm [Yu 00], yet we show that higher compression does not necessarily lead to an improvement in segmentation accuracy, as we improve on the reference segmentation lengths throughout all experimental datasets.

\section{Proposed Method}

\section{$3 \cdot 1$ Unsupervised algorithm}

\section{$\S 1$ The MDL principle}

The minimum description length principle for model selection and inductive inference was introduced by Rissanen [Rissanen 78]. In this approach to statistical modeling, the compression of the observed data achieved by the considered hypothesis is regarded as a basic criterion for their evaluation, as it reflects the extent to which they capture the regularity present in the data. The learning conducted in this way implies a trade-off between the goodness-offit to the data and the complexity of the model, and ensures that in spite of its richness, the model will retain a high predictive capability. Among the applications of MDL in natural language processing other than word segmentation, hereby we mention grammar induction [Adriaans 06], word clustering [Li 98], morphological analysis 
[Creutz 02], transliteration [Zelenko 09], case frame analysis [Li 98] and query analysis [Cao 08].

MDL is closely related to Bayesian maximum a posteriori (MAP) inference. Depending on the choice of a universal code, the two approaches can overlap, as is the case with the two-part code discussed in this paper. It can be shown that the model selection in our method is equivalent to a MAP inference conducted under the assumption that the prior probability of a model decreases exponentially with its length.

\section{$\S 2$ Word segmentation with MDL}

The proposed two-part code incorporates some extensions of models presented in related work, aimed at achieving a more precise estimation of the representation length.

We first introduce the general two-part code, which consists of:

- the model, embodied by a codebook, i.e., a lexicon of unique word types $M=\left\{w_{1}, \ldots, w_{|M|}\right\}$,

- the source text $D$, obtained through encoding the corpus using the lexicon.

The total description length amounts to the number of bits necessary for simultaneous transmission of the codebook and the source text. Therefore, our objective is to minimize the combined description length of both terms:

$$
L(D, M)=L(M)+L(D \mid M) .
$$

The description length of the data given $M$ is calculated using the Shannon-Fano code:

$$
L(D \mid M)=-\sum_{j=1}^{|M|} \# w_{j} \log _{2} P\left(w_{j}\right),
$$

where $\# w_{j}$ stands for the frequency of the word $w_{j}$ in the text.

Different strategies have been proposed for the calculation of the codebook cost. A common technique in segmentation and morphology induction models is to calculate the product of the length in characters of the lexicon and an estimate of the per-character entropy. In this way, both the probabilities and lengths of words are taken into consideration. The use of a constant value is an effective and easily computable approach, but it is far from precise. For instance, in [Yu 00] the average entropy per character is measured against the original corpus, but this model does not capture the effects of the word distributions on the observed character probabilities. For this reason, we propose a different method: the codebook is modeled as a separate Markov chain of characters.

A lexicon of characters $M^{\prime}$ is defined. The description length of the lexicon data $D^{\prime}$ given $M^{\prime}$ is then calculated as:

$$
L\left(D^{\prime} \mid M^{\prime}\right)=-\sum_{i=1}^{|C|} \# c_{i} \log _{2} P\left(c_{i}\right)
$$

where $\# c_{i}$ denotes the frequency of a character $c_{i}$ in the lexicon of hypothesis $M$. The term $L\left(M^{\prime}\right)$ is constant for any choice of hypothesis, as it represents the character set of a corpus.

The total description length under the proposed model is calculated as:

$$
\begin{gathered}
L(M)+L(D \mid M) \\
=L\left(M^{\prime}\right)+L\left(D^{\prime} \mid M^{\prime}\right)+L(D \mid M) \\
=-\sum_{i=1}^{|C|} \# c_{i} \log _{2} P\left(c_{i}\right)-\sum_{j=1}^{|M|} \# w_{j} \log _{2} P\left(w_{j}\right)+O(1) .
\end{gathered}
$$

A rigorous definition of the description length should include two additional terms, $L(\theta \mid M)$ and $L\left(\theta^{\prime} \mid M^{\prime}\right)$, which give the representation cost of the parameters of both models. The term $L(\theta \mid M)$ can be calculated as:

$$
L(\theta \mid M)=\frac{|M|-1}{2} * \log _{2} S,
$$

where $|M|-1$ gives the number of parameters (degrees of freedom), and $S$ is the size of the dataset (the total length of the text in characters). The parametric complexity term for the lexicon is calculated analogously. For a derivation of the above formula, refer to Li's thesis [Li 98].

The MDL framework does not provide standard search algorithms for obtaining the hypotheses that minimize the description length. In the rest of this section, we will introduce an efficient technique suitable for the word segmentation task.

\section{$\S 3$ Obtaining an initial hypothesis}

First, a rough initial hypothesis is built by an algorithm that combines the branching entropy and MDL criteria.

Given a set $\mathcal{X}$, comprising all the characters found in a text, the entropy of branching at position $k$ of the text is defined as:

$$
\begin{aligned}
& H\left(X_{k} \mid x_{k-1}, \ldots, x_{k-n}\right) \\
= & -\sum_{x \in \mathcal{X}} P\left(x \mid x_{k-1}, \ldots, x_{k-n}\right) \log _{2} P\left(x \mid x_{k-1}, \ldots, x_{k-n}\right),
\end{aligned}
$$

where $x_{k}$ represents the character found at position $k$, and $n$ is the order of the Markov model over characters. For brevity, hereafter we shall denote the observed sequence $\left\{x_{k-1}, \ldots, x_{k-n}\right\}$ as $\left\{x_{k-1: k-n}\right\}$.

The above definition is extended to combine the entropy estimates in the left-to-right and right-to-left directions, as this factor has reportedly improved performance figures 
for models based on branching entropy [Jin 06]. The estimates in both directions are summed up, yielding a single value per position:

$$
\begin{aligned}
& H^{\prime}\left(X_{k ; k-1} \mid x_{k-1: k-n} ; x_{k: k+n-1}\right) \\
= & -\sum_{x \in \mathcal{X}} P\left(x \mid x_{k-1: k-n}\right) \log _{2} P\left(x \mid x_{k-1: k-n}\right) \\
& -\sum_{x \in \mathcal{X}} P\left(x \mid x_{k: k+n-1}\right) \log _{2} P\left(x \mid x_{k: k+n-1}\right) .
\end{aligned}
$$

Suffix arrays are employed during the collection of frequency statistics. For a character model of order $n$ over a testing corpus of size $t$ and a training corpus of size $m$, suffix arrays allow these to be acquired in at most $O(t n \log m)$ time. Faster implementations reduce the complexity to $O(t(n+\log m))$. For further discussion, see [Manber 91].

The chunking technique we adopt is to insert a boundary when the branching entropy measured in sequences of length $n$ exceeds a threshold $\beta\left(H\left(X \mid x_{k-1: k-n}\right)>\beta\right)$. Both $n$ and $\beta$ are fixed.

Since the F-score curve obtained as decreasing values are assigned to the threshold is typically unimodal as in many applications of MDL, we employ a bisection search routine for the estimation of the threshold (Algorithm 1).

All positions of the dataset are sorted by their entropy values. At each iteration, at most two new hypotheses are built, and their description lengths are calculated in time linear to the data size. The computational complexity of the described routine is $O(t \log t)$, where $t$ is the corpus length in characters. Since the collection of frequency statistics requires the computation of $O(t n \log m)$, the total computational complexity for obtaining an initial hypothesis is $O(t n \log m+t \log t)$.

The order of the Markov chain $n$ used during the entropy calculation is the only input variable of the proposed model. Since different values perform best across various languages, the most appropriate settings can be obtained with the help of a small annotated corpus. However, the MDL objective also enables the conduction of unsupervised optimization against sufficiently large unlabeled datasets. The order that minimizes the description length of the data can be discovered in a few iterations of Algorithm 1 with increasing values of $n$, and it typically matches the optimal value of the parameter (Table 1).

Although an acceptable initial segmentation can be built using the described approach, it is possible to obtain a higher F-score with an extended model that takes into account the statistics of Markov chains from several orders during the entropy calculation. This can be done by summing up the entropy estimates, in the way introduced ear-

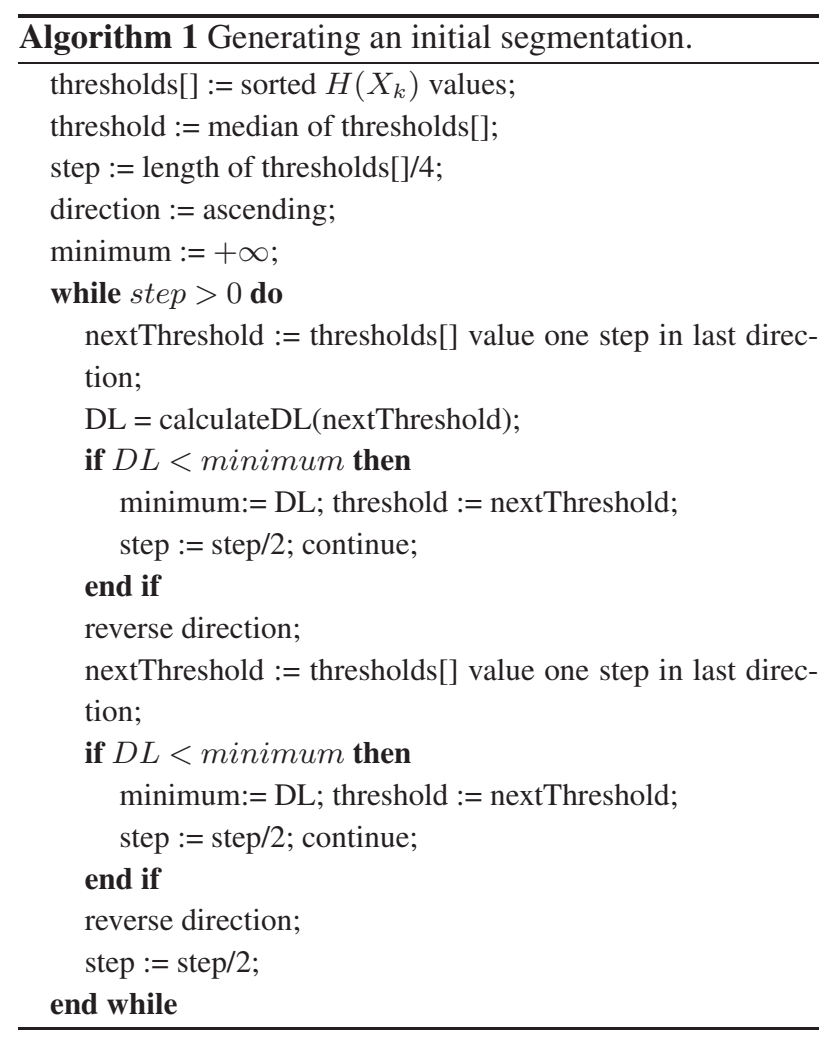

Table 1: Length in bits of the solutions proposed by Algorithm 1 with respect to the character $\mathrm{n}$-gram order.

\begin{tabular}{l||l|l|l|l}
\hline Corpus & 1-gram & 2-gram & 3-gram & 4-gram \\
\hline \hline CHILDES $\left(\times 10^{5}\right)$ & 3.947 & $\mathbf{3 . 6 7 7}$ & 3.681 & 4.053 \\
\hline Kyoto $\left(\times 10^{7}\right)$ & 1.291 & $\mathbf{1 . 2 8 9}$ & 1.398 & 1.837 \\
\hline
\end{tabular}

lier for combining the values in both directions:

$$
\begin{aligned}
& H^{\prime \prime}\left(X_{k ; k-1} \mid x_{k-1: k-n} ; x_{k: k+n-1}\right) \\
=- & \sum_{n=1}^{n_{\max }}\left(\sum_{x \in \mathcal{X}} P\left(x \mid x_{k-1: k-n}\right) \log _{2} P\left(x \mid x_{k-1: k-n}\right)\right. \\
& \left.+\sum_{x \in \mathcal{X}} P\left(x \mid x_{k: k+n-1}\right) \log _{2} P\left(x \mid x_{k: k+n-1}\right)\right),
\end{aligned}
$$

where $n_{\max }$ is the index of the highest order to be taken into consideration.

\section{$\S 4$ Refining the initial hypothesis}

In the second phase of our algorithm, we will refine the initial hypothesis through the reorganization of local cooccurrences which produce redundant description length.

Among the variety of possible approaches, we opt for greedy optimization, as our primary interest is to further explore the impact that description length minimization has on segmentation performance. Of course, such an approach is unlikely to obtain global minima, but it is a feasible means of conducting the optimization process, and guarantees a certain increase in compression.

Since a preliminary segmentation is available, it is convenient to proceed by inserting or removing boundaries in 
the text, thus splitting or merging the already discovered tokens. The ranked positions involved in the previous step can be reused here, as this is a way to bias the search towards areas of the text where boundaries are more likely to occur. Boundary insertion should start in regions where the branching entropy is high, and removal should first occur in regions where the entropy is close to zero. A drawback of this approach is that it omits locations where the gains are not immediately obvious, as it cannot assess the cumulative gains arising from the merging or splitting of all occurrences of a certain pair (Algorithm 2).

A clean-up routine, which compensates for this shortage is also implemented (Algorithm 3). It operates directly on the types found in the lexicon produced by Algorithm 2, and is capable of modifying a large number of occurrences of a given pair in a single step. The lexicon types are sorted by their contribution to the total description length of the corpus. For each word type, splitting or merging is attempted at every letter, beginning from the center. The algorithm eliminates unlikely types with low contribution, which represent mostly noise, and redistributes their cost among the more likely ones. The design of the merging routine makes it impossible to produce types longer than the ones already found in the lexicon, as an exhaustive search would be computationally prohibitive.

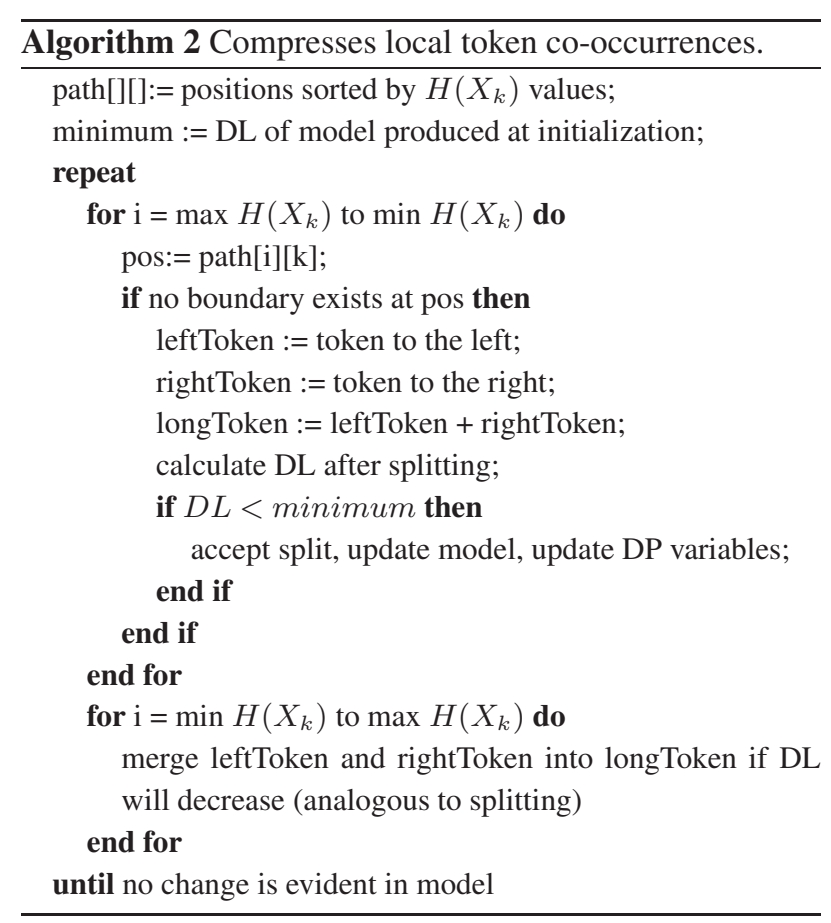

The evaluation of each hypothetical change in the segmentation requires that the description length of the twopart code is recalculated. In order to make this optimization phase computationally feasible, a dynamic programming technique is employed in Algorithms 2 and 3. The

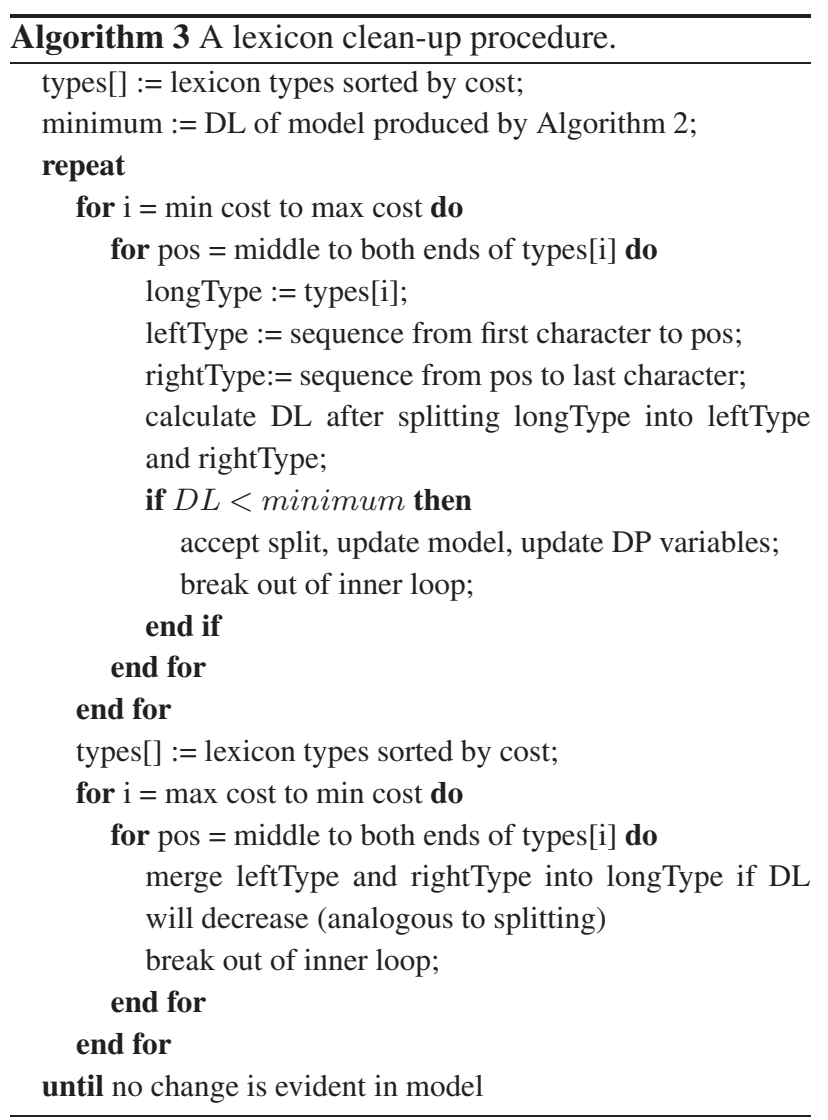

approach adopted for the recalculation of the source text term $L(D \mid M)$ is explained below. The estimation of the lexicon cost is analogous. The term $L(D \mid M)$ can be rewritten as:

$$
\begin{gathered}
L(D \mid M)=-\sum_{j=1}^{|M|} \# w_{j} \log _{2} \frac{\# w_{j}}{N} \\
=-\sum_{j=1}^{|M|} \# w_{j} \log _{2} \# w_{j}+N \log _{2} N=T_{1}+T_{2},
\end{gathered}
$$

where $\# w_{j}$ is the frequency of $w_{j}$ in the segmented corpus, and $N=\sum_{j=1}^{|M|} \# w_{j}$ is the cumulative token count. In order to calculate the new length, we keep the values of the terms $T_{1}$ and $T_{2}$ obtained at the last change of the model. Their new values are computed for each hypothetical split or merge on the basis of the last values, and the expected description length is calculated as their sum. If the produced estimate is lower, the model is modified and the new values of $T_{1}$ and $T_{2}$ are stored for future use.

In order to maintain precise token counts, Algorithms 2 and 3 recognize the fact that recurring sequences ("byebye", etc.) appear in the corpora, and handle them accordingly. Known boundaries, such as the sentence boundaries in the CHILDES corpus, are also taken into consideration.

The theoretical computational complexity of Algorithms 2 and 3 cannot be estimated. In practice, however, the outer loops of these algorithms usually repeat only a few 
times. For example, Algorithm 2 converged after 6 iterations and Algorithm 3 converged after 5 iterations in the later experiment with MSR, which is the dataset with the largest number of tokens (Table 2).

\section{$\S 5$ Segmentation of standalone datasets via a trained model}

The unsupervised method discussed until this point induces the lexicon of a sample of textual data by means of probabilistic inference. Naturally, the accuracy of results tends to decrease when the provided language samples are small. In order to guarantee that the highest possible evaluation scores are achieved consistently, we introduce a process through which training with an arbitrary amount of text can be conducted prior to the segmentation of standalone experimental data. In this way, more control can be exerted on the performance of the model through the provision of relevant training data, including its optimization for particular domains.

During the training phase, a large unlabeled corpus is fed into the algorithm, and a language model is obtained by means of description length minimization (Algorithms 1-3). During the segmentation phase, the algorithm updates the trained model as it builds the segmentation hypothesis for the previously unseen data. The objective in this case is to minimize the total representation cost of the training and the experimental data.

\section{$3 \cdot 2$ Semi-supervised word segmentation}

\section{$\S 1$ Motivation}

Various natural language processing techniques of continuous writing scripts depends greatly on the correctness of segmentation, as errors are propagated to all subsequent levels of analysis (POS tagging, dependency parsing, knowledge extraction, etc.). A major challenge in supervised word segmentation is to ensure the robustness of the model with respect to previously unencountered sequences, such as out-of-vocabulary words and highly ambiguous context. As languages evolve over time, it is impossible to achieve consistent results with an invariable supervised model. Moreover, even elaborate models tend to lose their accuracy when processing out-of-domain data.

The unsupervised algorithm discussed in Section 3.1 is a relatively inexpensive and highly accurate heuristic approach for word discovery that adapts well to different languages and domains. However, it is not always a practical approach to word segmentation on its own. Segmentation standards can vary depending on the final task, and therefore labeled examples are mandatory for achieving a satisfactory performance. Furthermore, supervised models tend to produce significantly lower error rates when sufficient training data is provided.

Ideally, we would like to make use of a hybrid technique that combines the strengths of the supervised and unsupervised approaches in order to obtain the highest possible performance. Such semi-supervised approaches should be able to take full advantage of the labeled data, and resort to heuristics when identifying unseen words and resolving ambiguities.

To our knowledge, the potential of unsupervised methods, equivalent to the state-of-the-art methods, to support semi-supervised word segmentation when trained with a large unlabeled dataset has not been assessed in previous work. In the following section, we formulate a simple semi-supervised framework that allows us to evaluate this aspect of the utility of the proposed algorithm.

\section{$\S 2$ Semi-supervised framework}

Conditional random fields (CRF), are a machine learning framework renowned for its performance in the labeling and segmentation of structured data [Lafferty 01]. Due to their discriminative nature, CRF achieve an unmatched accuracy in capturing arbitrary, non-independent relationships among the observation sequences. The model relaxes the strong independence assumptions found in conventional generative models, and therefore can estimate the hidden state probabilities with regard to past and future observations. Furthermore, CRF resolve the "label bias" problem found in other non-generative models, such as Maximum Entropy Markov Models [McCallum 00]. These properties motivate us to choose $\mathrm{CRF}$ as the foundation of our semi-supervised word segmentation framework.

We formulate a model that operates on the character level. Although methods based on word lattices are more common in practice due to their greater speed, characterbased models are straightforward to implement, and can achieve a higher accuracy [ $\mathrm{Ng} \mathrm{04],} \mathrm{which} \mathrm{is} \mathrm{an} \mathrm{important}$ factor in this preliminary evaluation. Our experimental model is restricted to the task of word segmentation, but it can be extended further to a full-featured POS-tagger through the techniques discussed in [ $\mathrm{Ng} \mathrm{04].}$

Our semi-supervised framework relies on the CRF mentioned above, but additionally incorporates the features derived via the proposed unsupervised method on top of a set of basic features for supervised learning, similar to the one described by in [Tsuboi 08]. Our feature set incorporates $n$-grams over characters and character classes, as well as features indicating the belonging of a given $n$-gram to a dictionary. However, instead of using external dictionaries, we match the $n$-grams against the lexicon induced by the unsupervised algorithm. Further details regarding the 
feature set are available in Section 4.3.

The major problems that need to be addressed in the semi-supervised setting pertain to the recognition of unknown words and the analysis of ambiguous context. To this end, we propose two types of features: lexical and contextual. The first type represents the results from looking up character $n$-grams in a lexicon, induced from a large amount of unlabeled data. The second type characterizes the context of a character, and includes the branching entropy values and the annotations proposed by the unsupervised algorithm.

The unlabeled data features include:

- the contribution of the types that correspond to the above defined character $n$-grams, as a percentage of the total description length of the induced model;

- the label predicted by the unsupervised algorithm for the current position;

- the branching entropy measured at the current position and the nearest predicted boundaries $\left(H^{\prime \prime}, n_{\max }=\right.$ $3)$.

We preferred to use the word's contribution to the model length instead of a binary attribute, as the contribution measure correlates with the probability of a word and the characters it incorporates. Branching entropy values are provided as an additional cue that can aid the resolution of ambiguities.

Those features are fed into a first-order Markov CRF.

\section{Experimental Settings}

\subsection{Evaluation measure}

We report the obtained F-score, precision and recall values. As precision and recall can be represented in terms of boundary, token or type counts, the scores are represented using each of these criteria. Precision $(P)$ and recall $(R)$ are defined as:

$$
P=\frac{\# \text { correct units }}{\# \text { output units }}, \quad R=\frac{\text { \#correct units }}{\# \text { gold standard units }} .
$$

Boundary, token and lexicon F-scores, denoted as $B-F$ and $T-F$ and $L-F$, are calculated as the harmonic averages of the corresponding precision and recall values.

\subsection{Unsupervised model}

We evaluated the proposed model on six datasets. The first one is the Bernstein-Ratner corpus for language acquisition based on transcripts from the CHILDES database [Ratner 87]. This dataset consists of phonetically transcribed utterances of adult speech directed to 13 through 21-month-old children. We evaluated the performance of our model in the cases when some of the boundaries among the individual sentences are available to it (B), and when it starts from a blank state $(\mathrm{N})$.

The Kyoto University Corpus [Kurohashi 98] is a standard dataset for Japanese morphological and dependency structure analysis, which comprises articles and editorials from the Mainichi Shimbun newspaper.

The BEST corpus for word segmentation and named entity recognition in Thai language combines text from a variety of sources including encyclopedia (E), newspaper articles $(\mathrm{N})$, scientific articles $(\mathrm{A})$, and novels $(\mathrm{F})$.

The WSJ subset of the Penn Treebank II Corpus incorporates selected stories from the Wall Street Journal, year 1989 [Marcus 94]. Both the original text (O), and a version in which all characters were converted to lower case (L) were used.

Two corpora contributed to the SIGHAN Bakeoff 2005 by the City University of Hong Kong (CITYU) and Microsoft Research, China (MSR) were used for evaluating the algorithm with Chinese language data [Emerson 05].

The datasets listed above were built by removing the tags and blank spaces found in the corpora, and concatenating the remaining text. We added two more training datasets for Japanese, which were used in a separate experiment solely for the acquisition of frequency statistics. One of them was created from 200,000 randomly chosen Wikipedia articles, stripped from structural elements. The other one contains text from the year 2005 issues of Asahi Newspaper. Statistics regarding all described datasets are presented in Table 2.

The entire corpora are segmented in each experiment. This is necessary for a direct comparison between the proposed model and the cited methods evaluated against the CHILDES corpus. The results presented for the Chinese language data have been calculated against the test splits made available to the participants in the SIGHAN Bakeoff, however the models have been trained using all available data, including the training and test splits of the corpora.

The system is implemented in Java with the suffix arrays through a C library called Sary. ${ }^{* 1}$ All experiments were conducted on a $2 \mathrm{GHz}$ Core2Duo T7200 machine with 2 GB RAM.

\section{$4 \cdot 3$ Semi-supervised model}

All corpora are preprocessed by the unsupervised model, in order to generate the state observations necessary for the operation of the CRF. We used CRF++ toolkit ${ }^{* 2}$ for training and inference of the CRF model. All features of

\footnotetext{
$* 1 \quad$ http://sary.sourceforge.net

$* 2$ http://crfpp.sourceforge.net
} 
Table 2: Corpora used during the evaluation. Precise token- and type counts have been omitted for Wikipedia and Asahi, as no gold standard segmentations are available.

\begin{tabular}{l||l|r|r|r|r}
\hline Corpus & Language & Size $(\mathrm{MB})$ & Chars $(\mathrm{K})$ & Tokens $(\mathrm{K})$ & Types (K) \\
\hline \hline CHILDES-B/N & English & 0.1 & 95.8 & 33.3 & 1.3 \\
\hline Kyoto & Japanese & 5.02 & 1674.9 & 972.9 & 39.5 \\
\hline WSJ & English & 5.22 & 5220.0 & 1174.2 & 49.1 \\
\hline BEST-E & Thai & 12.64 & 4360.2 & 1163.2 & 26.2 \\
\hline BEST-N & Thai & 18.37 & 6422.7 & 1659.4 & 36.3 \\
\hline BEST-A & Thai & 4.59 & 1619.9 & 438.7 & 13.9 \\
\hline BEST-F & Thai & 16.18 & 5568.0 & 1670.8 & 22.6 \\
\hline CITYU & Chinese (Traditional) & 8.38 & 2471.0 & 1496.6 & 70.8 \\
\hline MSR & Chinese (Simplified) & 16.8 & 4234.9 & 2475.3 & 90.1 \\
\hline \hline Wikipedia & Japanese & 425.0 & 169069.3 & & $/$ \\
\hline Asahi & Japanese & 337.2 & 112401.1 & & $/$ \\
\hline BEST-All & Thai & 51.2 & 17424.0 & 4371.8 & 73.4 \\
\hline
\end{tabular}

real values undergo normalization and discretization, and are rendered as integers in the range between 0 and 10. A special character is reserved for unknown values.

No external dictionaries are accessed by the proposed model. The feature set for supervised learning includes the character and character class $n$-grams (with $n=1,2,3$ ) on both sides of the current position. The following character classes are defined: ASCII Latin letters, ASCII numerals, ASCII symbols, hiragana, katakana, half width katakana, full width Latin letters, full width numerals, kanji, symbols.

The semi-supervised algorithm also requires that features from the unsupervised boundary learning method are provided. These include: 1 . the boundary predictions of the unsupervised algorithm (a binary feature that denotes the presence or absence of a boundary at the current character); 2. the branching entropy values measured at the current character and the two characters that surround it; all branching entropy values are re-scaled with respect to the minimal and maximal values observed throughout the entire corpus, and are consequently discretized to integer values that lie in the range between 0 and $10 ; 3$. the contributions of the $n$-grams (with $n=1,2,3$ ) on both sides of the current position to the model in terms of the quantity $L(w, M)$, where $w$ is a type belonging to the induced lexicon; these quantities are re-scaled with respect to the minimal and maximal contributions observed for any type belonging to the model, and are consequently discretized to integer values that lie in the range between 0 and 10 .

The segmentation performance of the semi-supervised model was evaluated in two series of experiments. In the first case only in-domain test data was used, in order to assess the validity of the proposed model and study the relationship between the amount of labeled training data and the achieved F-score. The latter experiment was focused
Table 3: Statistics for the KNBC corpus. Out-of-vocabulary (OOV) word token and type counts are calculated with respect to Kyoto corpus.

\begin{tabular}{l|l|l|l|l|l}
\hline $\begin{array}{l}\text { Size } \\
(\mathrm{MB})\end{array}$ & Chars & Tokens & Types & $\begin{array}{l}\text { OOV To- } \\
\text { kens }(\%)\end{array}$ & $\begin{array}{l}\text { OOV Ty- } \\
\text { pes }(\%)\end{array}$ \\
\hline \hline 0.437 & 123226 & 66952 & 8527 & $\begin{array}{l}4858 \\
(7.26 \%)\end{array}$ & $\begin{array}{l}2616 \\
(30.68 \%)\end{array}$ \\
\hline
\end{tabular}

on the problem of domain adaptation, and we employed an out-of-domain test dataset.

The first group of experiments was conducted using Kyoto corpus as a source of labeled data. For each trial, we generated a test dataset by concatenating approximately $10 \%$ of the articles found in the corpus, chosen at random (sizes varied slightly due to the concatenation of whole articles). We generated training datasets of different sizes $(0.1 \%-90 \%)$ from the remaining data through the same process, truncating some articles in order to obtain the smallest training datasets. This method for experimental data construction allows a strict evaluation, and guarantees that no overlapping occurs between the training and test data. We carried out five trials per experiment, with new test and training datasets for every trial.

For unsupervised training we employed a $50 \mathrm{MB}$ subset of the Asahi corpus. We calculated entropy using frequency statistics from the entire Asahi corpus.

In the domain adaptation experiment, labeled data came from the Kyoto corpus, and the semi-supervised model was evaluated against the $K N B C$ corpus of labeled blog data*3. The unsupervised algorithm was trained using a $50 \mathrm{MB}$ collection of unlabeled blog data. HTML tags in the unlabeled data were regarded as known boundaries, in order to reduce the errors as much as possible. We computed entropy with frequency statistics from a $400 \mathrm{MB}$ collection of unlabeled blog data.

*3 http://nlp.kuee.kyoto-u.ac.jp/NLP_Portal/jeita_corpus/index.html 
Table 5: Comparison of the proposed method (2a, 2b) with the model of [Jin 06], referred to as JT in the table. Unigrams to 6-grams are used in JT. Execution times (seconds) include the obtaining of frequency statistics, and are represented by averages over 10 runs. DL indicates description length measured in bits. Ref.DL indicates description length of reference segmentation.

\begin{tabular}{|c|c|c|c|c|c|c|c|c|c|c|}
\hline Model & Corpus \& Settings & B-Prec & B-Rec & B-F & T-Prec & T-Rec & T-F & DL & Ref.DL & Time \\
\hline 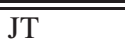 & 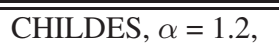 & "0.8667 & "0.8898 & (2.8781 & 0.6808 & 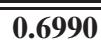 & 0.6898 & $3.448 \times 10^{5}$ & \multirow{3}{*}{$3.005 \times 10^{5}$} & 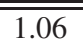 \\
\hline $2 \mathrm{a}\left(H^{\prime}\right)$ & CHILDES, $n=2$ & 0.7636 & 0.9109 & 0.8308 & 0.5352 & 0.6384 & 0.5823 & $3.677 \times 10^{5}$ & & 0.75 \\
\hline $2 \mathrm{~b}\left(H^{\prime \prime}\right)$ & CHILDES, $n_{\max }=3$ & 0.8692 & 0.8865 & 0.8777 & 0.6792 & 0.6927 & 0.6859 & $3.476 \times 10^{5}$ & & 0.89 \\
\hline$\overline{\mathrm{JTT}}$ & Kyoto, $\alpha=0$, & $\overline{0.8208}$ & 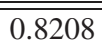 & 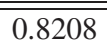 & 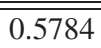 & 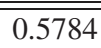 & 0.5784 & $1.325 \times 10^{7}$ & \multirow{3}{*}{$1.120 \times 10^{7}$} & 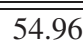 \\
\hline $2 \mathrm{a}\left(H^{\prime}\right)$ & Kyoto, $n=2$ & 0.8100 & 0.8621 & 0.8353 & 0.5934 & 0.6316 & 0.6119 & $1.289 \times 10^{7}$ & & 22.91 \\
\hline $2 \mathrm{~b}\left(H^{\prime \prime}\right)$ & Kyoto, $n_{\max }=2$ & 0.8024 & 0.9177 & 0.8562 & 0.6093 & 0.6969 & 0.6501 & $1.248 \times 10^{7}$ & & 23.21 \\
\hline
\end{tabular}

Table 4: Coverage of the lexicons induced from the unlabeled corpora with respect to $\mathrm{OOV}$ in $\mathrm{KNBC}$.

\begin{tabular}{l|l|l|l}
\hline $\begin{array}{l}\text { Unlabeled } \\
\text { Corpus }\end{array}$ & $\begin{array}{l}\text { Size } \\
(\mathrm{MB})\end{array}$ & $\begin{array}{l}\text { Induced } \\
\text { Types }\end{array}$ & $\begin{array}{l}\text { KNBC OOV } \\
\text { Types (\%) }\end{array}$ \\
\hline \hline Asahi & 50.0 & 55088 & $514(0.20 \%)$ \\
\hline Blog Data & 50.0 & 151249 & $872(0.33 \%)$ \\
\hline
\end{tabular}

The reason for choosing the Kyoto and KNBC combination is two-fold. Firstly, the KNBC annotations follow the same standard as Kyoto corpus, and this eliminates the errors due to annotator disagreement. Secondly, blog data is particularly difficult to handle without a domain adaptation mechanism. In contrast to the vocabulary found in newspaper articles, blog posts are characterized by a wide use of informality, abbreviations and acronyms, pseudographic patterns of various kinds and complicity, as well as a higher incidence of misspelling. Blog data is plentiful and easily obtainable, and thus allows us to assess the usefulness of the proposed word induction method as a supporting technique in domain adaptation.

Statistics regarding the unknown words in KNBC with respect to Kyoto corpus are presented in Table 3. The coverage of the lexicons induced from the $50 \mathrm{MB}$ Asahi and blog datasets with respect to the unknown words in KNBC are presented in Table 4. The blog data corpus gives rise to a particularly varied lexicon, as it includes quite noisy data (some Chinese and English text was found among the Japanese blog posts). However, both lexicons seem to include much of the vocabulary specific to $\mathrm{KNBC}$, and therefore have potential to improve the performance of labeling.

\section{Results and Discussion}

\section{$5 \cdot 1$ Unsupervised model}

The scores we obtained using the different instantiations of the branching entropy criterion at the initialization phase are displayed in Table 5, along with those generated by our implementation of the method presented in [Jin 06], where the threshold parameter $\alpha$ has been adjusted manually for optimal performance.
The experimental results are summarized in Tables 6 and 7. Durations include the obtaining of frequency statistics. The $n_{\max }$ parameter is set to the value which maximizes the compression during the initial phase, in order to make the results representative of the case in which no annotated development corpora are accessible to the algorithm.

It is evident that after the optimization carried out in the second phase, the description length is reduced to levels significantly lower than the ground truth. In this aspect, the algorithm outperforms the EM-based method of [Yu 00].

We conducted experiments involving various initialization strategies: scattering boundaries at random throughout the text, starting from entirely unsegmented state, or considering each symbol of the text to be a separate token. The results obtained with random initialization confirm the strong relationship between compression and segmentation accuracy, evident in the increase of token F-score between the random initialization and the termination of the algorithm, where description length is lower (Table 8). They also reveal the importance of the branching entropy criterion to the generation of hypotheses that maximize the evaluation scores and compression, as well as the role it plays in the reduction of computational time.

The greedy algorithms failed to suggest any optimizations that improve the compression in the cases when the boundaries/character ratio is either 0 or 1 . When no boundaries are given, splitting operations produce unique types with a low frequency that increase the cost of both parts of the MDL code, and are rejected. The algorithm runs slowly, as each evaluation operates on candidate strings of enormous length. Similarly, when the corpus is broken down into single-character tokens, merging individual pairs does not produce any increase in compression. This could be achieved by an algorithm that estimates the total effect from merging all instances of a given pair, but such an approach would be computationally infeasible for large corpora.

Finally, we tried randomizing the search path for Algo- 
Table 6: Results obtained after the termination of Algorithm 3.

\begin{tabular}{l||l|l|l|l}
\hline Corpus \& Settings & B-F & T-F & L-F & Time (s) \\
\hline \hline $\begin{array}{l}\text { CHILDES-B, } \\
n_{\max }=3\end{array}$ & 0.9092 & 0.7542 & 0.5890 & 2.60 \\
\hline $\begin{array}{l}\text { CHILDES-N, } \\
n_{\max }=3\end{array}$ & 0.9070 & 0.7499 & 0.5578 & 2.95 \\
\hline Kyoto, $n_{\max }=2$ & 0.8855 & 0.7131 & 0.3725 & 70.17 \\
\hline BEST-E, $n_{\max }=5$ & 0.9081 & 0.7793 & 0.3549 & 738.06 \\
\hline BEST-N, $n_{\max }=5$ & 0.8811 & 0.7339 & 0.2807 & 505.33 \\
\hline BEST-A, $n_{\max }=5$ & 0.9045 & 0.7632 & 0.4246 & 250.86 \\
\hline BEST-F, $n_{\max }=5$ & 0.9343 & 0.8216 & 0.4820 & 305.52 \\
\hline WSJ-O, $n_{\max }=6$ & 0.8405 & 0.6059 & 0.3338 & 658.21 \\
\hline WSJ-L, $n_{\max }=6$ & 0.8515 & 0.6373 & 0.3233 & 582.38 \\
\hline CITYU, $n_{\max }=4$ & 0.9143 & 0.7788 & 0.6993 & 86.53 \\
\hline MSR, $n_{\max }=3$ & 0.9247 & 0.8133 & 0.6706 & 128.62 \\
\hline
\end{tabular}

Table 7: Description length - proposed versus reference segmentation (entire datasets, including test splits, where available).

\begin{tabular}{l|c|c}
\hline \multirow{2}{*}{ Corpus \& Settings } & \multicolumn{2}{|c}{ Description Length } \\
\cline { 2 - 3 } & proposed & reference \\
\hline \hline CHILDES-B, $n_{\max }=3$ & $\mathbf{2 . 9 0 6} \times \mathbf{1 0}^{\mathbf{5}}$ & $3.005 \times 10^{5}$ \\
\hline CHILDES-N, $n_{\max }=3$ & $\mathbf{2 . 9 0 7} \times \mathbf{1 0}^{\mathbf{5}}$ & $3.005 \times 10^{5}$ \\
\hline Kyoto, $n_{\max }=2$ & $\mathbf{1 . 0 7 8} \times \mathbf{1 0}^{\mathbf{7}}$ & $1.120 \times 10^{7}$ \\
\hline BEST-E, $n_{\max }=5$ & $\mathbf{1 . 1 8 0} \times \mathbf{1 0}^{\mathbf{7}}$ & $1.252 \times 10^{7}$ \\
\hline BEST-N, $n_{\max }=5$ & $\mathbf{1 . 6 7 0} \times \mathbf{1 0}^{\mathbf{7}}$ & $1.809 \times 10^{7}$ \\
\hline BEST-A, $n_{\max }=5$ & $\mathbf{4 . 4 3 9} \times \mathbf{1 0}^{\mathbf{6}}$ & $4.711 \times 10^{6}$ \\
\hline BEST-F, $n_{\max }=5$ & $\mathbf{1 . 5 6 2} \times \mathbf{1 0}^{\mathbf{7}}$ & $1.634 \times 10^{7}$ \\
\hline WSJ-O, $n_{\max }=6$ & $\mathbf{1 . 3 5 8} \times \mathbf{1 0}^{\mathbf{7}}$ & $1.460 \times 10^{7}$ \\
\hline WSJ-L,,$n_{\max }=6$ & $\mathbf{1 . 3 1 7} \times \mathbf{1 0}^{\mathbf{7}}$ & $1.399 \times 10^{7}$ \\
\hline CITYU, $n_{\max }=4$ & $\mathbf{1 . 8 8 7} \times \mathbf{1 0}^{\mathbf{7}}$ & $1.974 \times 10^{7}$ \\
\hline MSR, $n_{\max }=3$ & $\mathbf{3 . 0 3 7} \times \mathbf{1 0}^{\mathbf{7}}$ & $3.177 \times 10^{7}$ \\
\hline
\end{tabular}

rithm 2 after an entropy-guided initialization, to observe a small deterioration in F-score in the final segmentation (less than $1 \%$ on average).

Figure 1(a) illustrates the effect that training data size has on F-score of segmentation for the Kyoto corpus. $100 \%$ for corpus size corresponds to $1674.9 \times 1000$ characters. The learning curves are similar throughout the different corpora. For the CHILDES corpus, which has a rather limited vocabulary, token F-score above $70 \%$ can be achieved for datasets as small as 5000 characters of training data, provided that reasonable values are set for the $n_{\max }$ parameter (we used the values presented in Table 7 throughout these experiments).

Table 8: Experimental results for CHILDES-N with randomized initialization and search path. The numbers in parentheses represent the seed boundaries/character ratios.

\begin{tabular}{l|l|l|l}
\hline \multicolumn{2}{c|}{ T-F-Score } & $\begin{array}{l}\text { Description } \\
\text { Length }\end{array}$ & $\begin{array}{l}\text { Time } \\
(\mathrm{s})\end{array}$ \\
\hline Random Init & Refinement & $3.876 \times 10^{5}$ & 6.66 \\
\hline $0.0441(0.25)$ & 0.3833 & $3.833 \times 10^{5}$ & 4.98 \\
\hline $0.0713(0.50)$ & 0.3721 & $4.127 \times 10^{5}$ & 3.75 \\
\hline $0.0596(0.75)$ & 0.2777 & & \\
\hline
\end{tabular}

Figure 1(b) shows the evolution of token F-score by stage for all corpora. The initialization phase seems to have the highest contribution to the formation of the final score, and the refinement phase is highly dependent on the output that it produces. As a consequence, results improve when a more adequate language sample is provided during the learning of local dependencies at initialization. This is evident in the experiments with the larger unlabeled Thai and Japanese corpora.

For Japanese language with the setting for the $n_{\text {max }}$ parameter that maximized compression, we observed an almost $4 \%$ increase in the token F-score produced at the end of the first phase with the Asahi corpus as training data. Only a small (less than $1 \%$ ) rise was observed in the overall performance. The quite larger dataset of randomly chosen Wikipedia articles achieved no improvement. We attributed this to the higher degree of correspondence between the domains of the Asahi and Kyoto corpora (Figure 1(c)).

Experiments with the BEST corpus provide a deeper insight into the influence of domain-specific data on the accuracy of segmentation. Performance deteriorates significantly when out-of-domain training data is used. In spite of its size, the assorted composite corpus, in which in-domain and out-of-domain training data are mixed, produces worse results than the corpora which include only domain-specific data (Figure 1(d)).

Finally, a comparison of our method with Bayesian ngram models is presented in Table 9. Through the increase of compression in the refinement phase of the algorithm, F-score is improved by around 3\%, and the scores approach those of the explicit probabilistic models of [Goldwater 06, Mochihashi 09]. It should be noticed that the figures of T-F on CHILDES in Table 9 fall short of that of the state-of-the-art; Johnson and Goldwater [Johnson 09] achieved 0.87 for T-F with the help of adaptor grammar. The proposed learner surpasses the other unsupervised word induction models in terms of processing speed. It should be noticed once again that different segmentation standards exist for Japanese, and therefore the "ground truth" provided by the Kyoto corpus cannot be considered an ideal measure of goodness of segmentation.

\section{$5 \cdot 2$ Semi-supervised model}

The averaged results from the closed evaluation are presented in Figure 2. The scores suggest that the effect of unlabeled data features on the performance becomes less expressed as more labeled data is provided to the model. Although the difference at the upper-bound F-score levels is negligible, the semi-supervised model consistently 


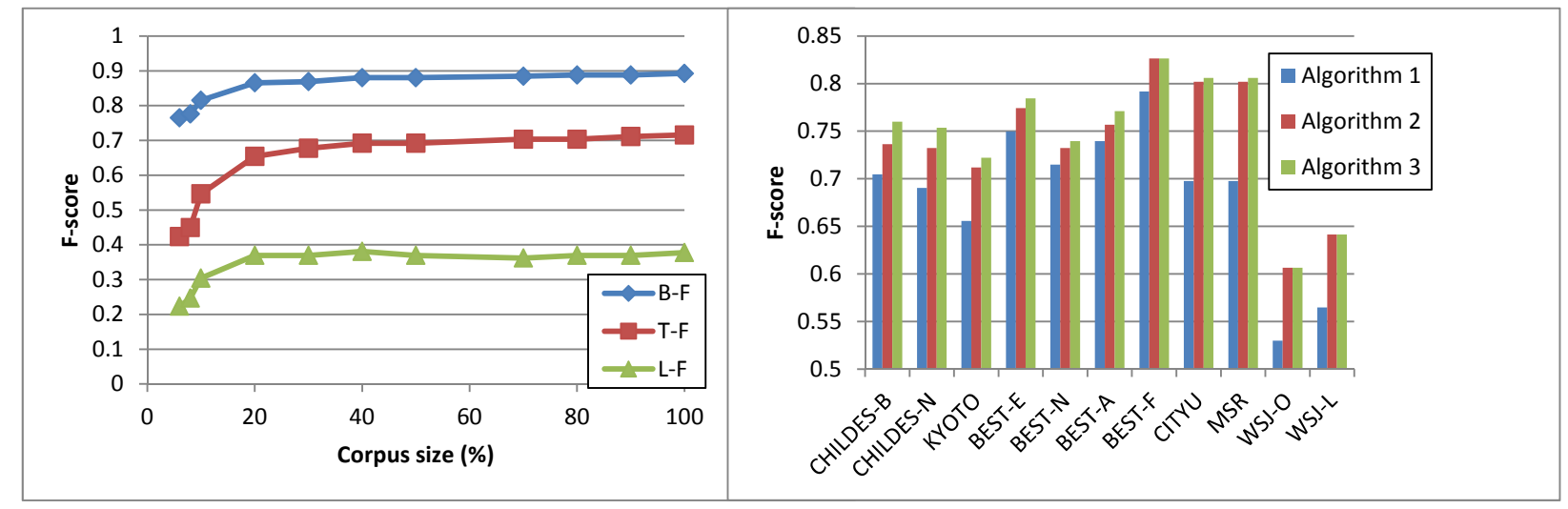

(a) corpus size/F-score relationship (Kyoto)

(b) F-score levels by phase

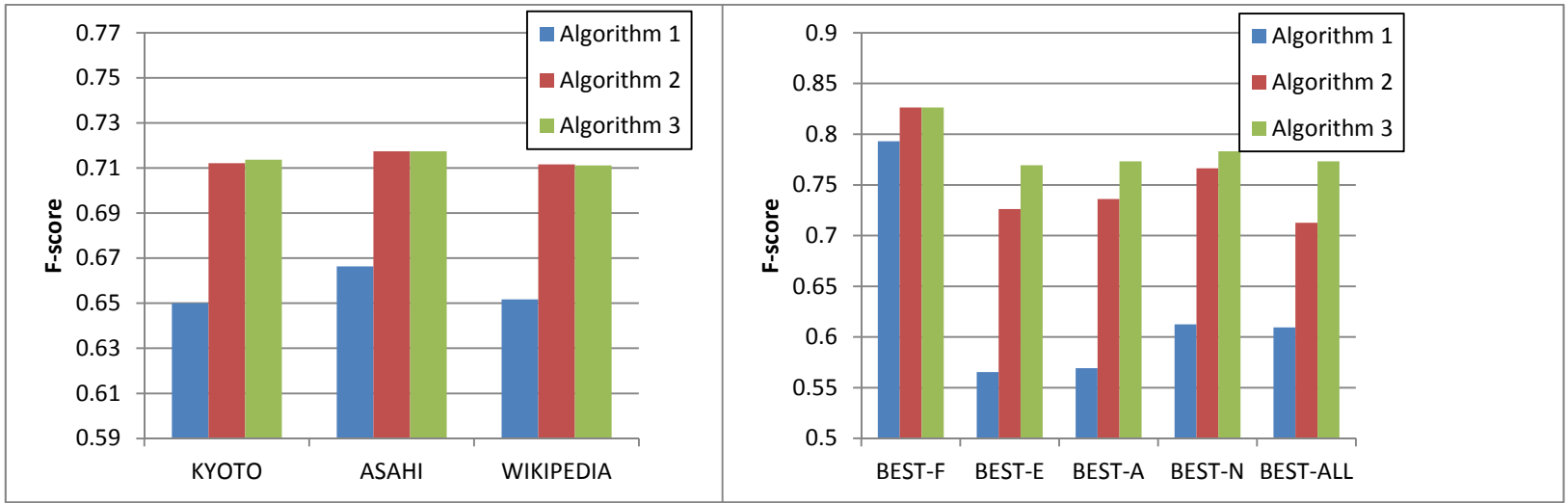

(c) F-score levels by phase with various Japanese corpora for frequency statistics (Kyoto)

(d) F-score levels by phase with various Thai corpora for frequency statistics (BEST)

Fig. 1: F-scores measured in various settings

outperforms the supervised one.

Further examination into recall values shown in Figures 3 and 4 reveals that the increase in F-score owes most to the improved recognition of known words. Significant error reduction for unknown words is also evident in the experiments with few labeled data. Unfortunately, this effect vanishes quickly, and unknown word recall deteriorates as the F-score improves. These observations point to an overfitting of the model to the training data, as unknown word instances are not encountered during the training phase. The performance can arguably be improved through the design of specific lexical features that match the $\mathrm{n}$-grams with the contents of an imperfect supervised lexicon, independent from the training data.

The contribution of the various types of unsupervised features was also evaluated. Our experiments have ranked the features that consult the induced lexicon as more important than the contextual features. Although costly, extensions such as the matching of longer n-grams and features that indicate the beginning or ending of a known lexicon word are likely to improve the performance. Our observations suggest that a model based on a word lattice could also benefit from the lexicons induced against unla-

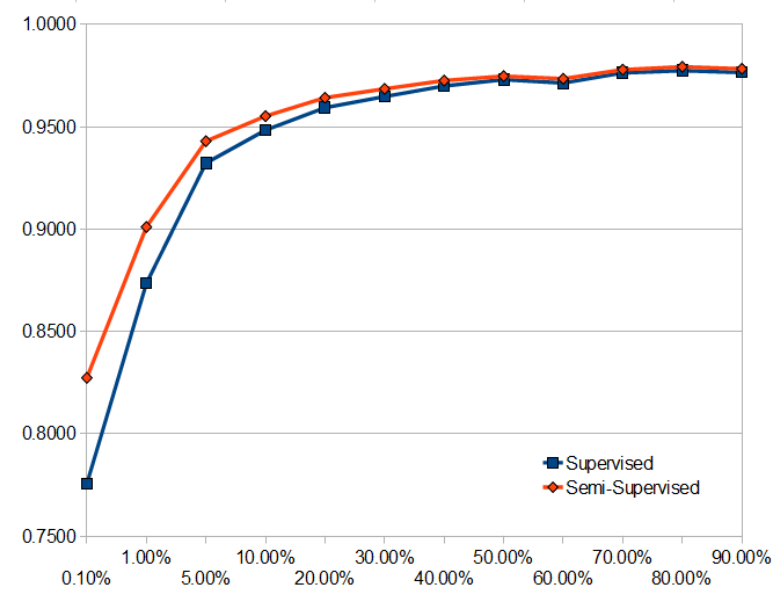

Fig. 2: F-score obtained by the supervised and semi-supervised models with different amounts of labeled training data from Kyoto corpus in a closed evaluation.

beled data.

The domain adaptation experiment reveals a similar rate of improvement. The well expressed decrease in F-score in comparison with the closed tests can be attributed to the high percentage of unknown words (Table 3). As can be seen on Table 11, the incorporation of unlabeled data leads 
Table 9: Comparison of the proposed method (Ent-MDL) with Mochihashi et al., 2009 (NPY) and Goldwater et al., 2006 (HDP). Results cited from Mochihashi et al. 2009.

\begin{tabular}{l|l|l|l|l|l|l|l|l}
\hline Model & Corpus & T-Prec & T-Rec & T-F & L-Prec & L-Rec & L-F & Time \\
\hline \hline NPY(3) & CHILDES & 0.7480 & 0.7520 & 0.7500 & 0.4780 & $\mathbf{0 . 5 9 7 0}$ & 0.5310 & 17 min \\
\hline NPY(2) & CHILDES & 0.7480 & $\mathbf{0 . 7 6 7 0}$ & $\mathbf{0 . 7 5 7 0}$ & 0.5730 & 0.5660 & 0.5700 & 17 min \\
\hline HDP(2) & CHILDES & 0.7520 & 0.6960 & 0.7230 & 0.6350 & 0.5520 & $\mathbf{0 . 5 9 1 0}$ & - \\
\hline Ent-MDL & CHILDES & $\mathbf{0 . 7 6 3 4}$ & 0.7453 & 0.7542 & $\mathbf{0 . 6 8 4 4}$ & 0.5170 & 0.5890 & $\mathbf{2 . 6 0}$ sec \\
\hline \hline NPY(2) & Kyoto & - & - & 0.6210 & - & - & - & - \\
\hline NPY(3) & Kyoto & - & - & 0.6660 & - & - & - & - \\
\hline Ent-MDL & Kyoto & 0.6912 & 0.7365 & $\mathbf{0 . 7 1 3 1}$ & 0.5908 & 0.2720 & 0.3725 & $70.16 \mathrm{sec}$ \\
\hline \hline NPY(2) & CITYU & - & - & $\mathbf{0 . 8 2 4 0}$ & - & - & - & - \\
\hline NPY(3) & CITYU & - & - & 0.8170 & - & - & - & - \\
\hline Ent-MDL & CITYU & 0.7703 & 0.7874 & 0.7788 & 0.7362 & 0.6659 & 0.6993 & $86.53 \mathrm{sec}$ \\
\hline \hline NPY(2) & MSR & - & - & 0.8020 & - & - & - & - \\
\hline NPY(3) & MSR & - & - & 0.8070 & - & - & - & - \\
\hline Ent-MDL & MSR & 0.7970 & 0.8303 & $\mathbf{0 . 8 1 3 3}$ & 0.7207 & 0.6270 & 0.6706 & $128.62 \mathrm{sec}$ \\
\hline \hline
\end{tabular}

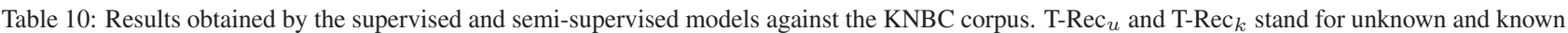
token recall, respectively.

\begin{tabular}{|c|c|c|c|c|c|c|c|c|c|c|}
\hline \multicolumn{5}{|c|}{ Supervised } & \multicolumn{5}{|c|}{ Semi-supervised } & \multirow{2}{*}{$\begin{array}{c}\text { Error } \\
\text { Reduction }\end{array}$} \\
\hline $\mathrm{T}-\mathrm{F}$ & T-Prec & T-Rec & T-Rec $u$ & T-Rec $k$ & T-F & T-Prec & T-Rec & $\mathrm{T}-\operatorname{Rec}_{u}$ & T-Rec ${ }_{k}$ & \\
\hline 0.9208 & 0.9212 & 0.9203 & 0.6639 & 0.9404 & 0.9268 & 0.9266 & 0.9270 & 0.6781 & 0.9465 & 0.0768 \\
\hline
\end{tabular}

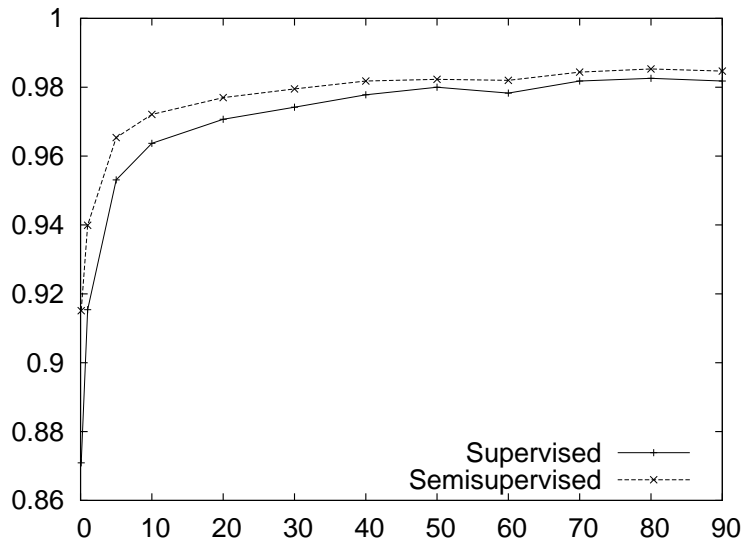

Fig. 3: Recall of known words

to a $1.5 \%$ increase in the recall for unknown tokens and $0.6 \%$ increase for known tokens. The total error reduction approaches $8 \%$.

It should be noticed that the blog data corpus comprises posts in Chinese and English that also appeared on the Japanese blogs. A more careful and targeted choice of data might lead to a higher lexicon accuracy, which could in turn improve the performance of the semi-supervised model.

In the domain adaptation experiment, we trained the unsupervised algorithm against a corpus of unlabeled blog articles that can be considered out-of-domain with respect to the Kyoto corpus. Preliminary evaluation has revealed that the performance of the semi-supervised method dete-

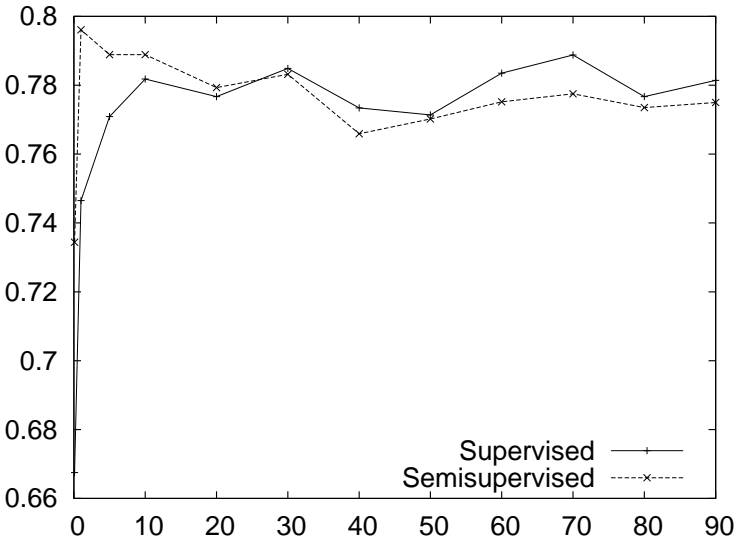

Fig. 4: Recall of unknown words

riorates significantly when different corpora are used for the annotation of the training and test data. If the domains of the labeled and unlabeled data are matched (Kyoto with Asahi, KNBC with the blog corpus), the performance drops below that of the supervised baseline. The results get even worse (nearly $2 \%$ decrease in token F-score) if blog data is used for annotating the Kyoto corpus, and Asahi for the KNBC corpus. The significant differences in the values of the observations when they are generated using two unlabeled datasets seem to impede the inference, and to exert a negative influence on segmentation performance. Alternatively, when we conducted the unsupervised training for both the training and test data with the Asahi corpus, we obtained a very modest improvement 
over the supervised baseline, as this setting did not provide access to any domain-specific data.

\section{Conclusions}

This paper has presented an efficient algorithm for unsupervised word induction, which relies on a combination of evidences. New instantiations of the branching entropy and MDL criteria have been proposed and evaluated against corpora in different languages. The MDLbased optimization eliminates the discretion in the choice of the context length and threshold parameters, common in segmentation models based on local statistics. At the same time, the branching entropy criterion enables a constrained search through the hypothesis space, allowing the proposed method to demonstrate a very high performance in terms of both F-score and speed.

A semi-supervised model that incorporates manual annotations and unlabeled data features has been designed and evaluated. Experiments have revealed that our framework can consistently improve the performance over a supervised baseline, and lead to a significant error reduction when very few labeled data is available. At the domain adaptation task, the semi-supervised word segmentation system has demonstrated an increase in the recall rates for both unknown and known word types, and higher overall F-score.

\section{Future Work}

Possible improvements of the proposed unsupervised method include modeling the dependencies among neighboring tokens, which would allow the evaluation of the context to be reflected in the cost function. Mechanisms for stochastic optimization implemented in the place of the greedy algorithms could provide an additional flexibility of search for such more complex models. Languagespecific rules can be incorporated into the inference procedures in order to reduce the error rates. As the proposed approach provides significant performance improvements, it could be utilized in the development of more sophisticated novel word induction schemes, e.g., ensemble models trained independently with different data.

The semi-supervised model presented in this paper consists of a very basic set of features, and is subject to numerous extensions. An appropriate starting point would be the introduction of novel lexical features to reduce the overfitting to the data and improve the recall with respect to unknown word types. An open test that relies on external lexical resources is also necessary. The effect of discretization error should be carefully assessed. A wordbased model should be considered as a more efficient combined approach to semi-supervised segmentation and morphological analysis. The domain adaptation experiments should be conducted with less noisy unlabeled data, and a fine-tuned version of the unsupervised algorithm that incorporates language- and domain-specific rules.

\section{Acknowledgment}

A preliminary version of this article was published in the proceedings of a conference [Zhikov 10]. A new idea and the related experiments that were not described in the preliminary version were added to this article.

The authors would like to thank the anonymous reviewers for their valuable comments and suggestions to improve the quality of the paper.

\section{$\diamond$ References $\diamond$}

[Adriaans 06] Adriaans, P. and Jacobs, C.: Using MDL for Grammar Induction, in Proceedings of the 8th International Colloquium on Grammatical Inference (Lecture Notes in Computer Science, vol.4201), pp. 293-306 (2006)

[Brent 96] Brent, M. R. and Cartwright, T. A.: Distributional Regularity and Phonotactic Constraints are Useful for Segmentation, Cognition, Vol. 61, No. 1-2, pp. 93-125 (1996)

[Cao 08] Cao, Y., Duan, H., Lin, C.-Y., Yu, Y., and Hon, H.-W.: Recommending questions using the mdl-based tree cut model, in Proceedings of the 17th international conference on World Wide Web, pp. 81-90 (2008)

[Creutz 02] Creutz, M. and Lagus, K.: Unsupervised discovery of morphemes, in Proceedings of the ACL-02 workshop on Morphological and phonological learning, pp. 21-30 (2002)

[Emerson 05] Emerson, T.: The second international Chinese word segmentation bakeoff, in Proceedings of the Fourth SIGHAN Workshop on Chinese Language Processing, pp. 123-133 (2005)

[Goldwater 06] Goldwater, S., Griffiths, T. L., and Johnson, M.: Contextual Dependencies in Unsupervised Word Segmentation, in Proceedings of the 21st International Conference on Computational Linguistics and the 44th Annual Meeting of the Association for Computational Linguistics, pp. 673-680 (2006)

[Goldwater 09] Goldwater, S., Griffiths, T. L., and Johnson, M.: A Bayesian Framework for Word Segmentation: Exploring the Effects of Context, Cognition, Vol. 112, No. 1, pp. 21-54 (2009)

[Harris 55] Harris, Z.: From Phoneme to Morpheme, Language, Vol. 31, No. 2, pp. 190-222 (1955)

[Huang 03] Huang, J. H. and Powers, D.: Chinese Word Segmentation Based on Contextual Entropy, in Proceedings of 17th Pacific Asia Conference on Language Information and Computation, pp. 152-158 (2003)

[Hutchens 98] Hutchens, J. L. and Alder, M. D.: Finding Structure via Compression, in Proceedings of the Conference on Computational Natural Language Learning, pp. 79-82 (1998)

[Jin 06] Jin, Z. and Tanaka-Ishii, K.: Unsupervised Segmentation of Chinese Text by Use of Branching Entropy, in Proceedings of the International Conference on computational Linguistics and the Annual Meeting of the Association for Computational Linguistics (Poster Session), pp. 428-435 (2006)

[Johnson 09] Johnson, M. and Goldwater, S.: Improving Nonparameteric Bayesian Inference: Experiments on Unsupervised Word Segmentation with Adaptor Grammars, in Proceedings of Human Language Technologies: The 2009 Annual Conference of the North 
American Association for Computational Linguistics, pp. 317-325 (2009)

[Kempe 99] Kempe, A.: Experiments in Unsupervised Entropy Based Corpus Segmentation, in Proceedings of the Conference on Computational Natural Language Learning, pp. 371-385 (1999)

[Kit 03] Kit, C.: How Does Lexical Acquisition Begin? A Cognitive Perspective, Cognitive Science, Vol. 1, No. 1, pp. 1-50 (2003)

[Kurohashi 98] Kurohashi, S. and Nagao, M.: Building a Japanese Parsed Corpus while Improving the Parsing System, in Proceedings of the First International Conference on Language Resources and Evaluation, pp. 719-724 (1998)

[Lafferty 01] Lafferty, J., McCallum, A., and Pereira, F.: Conditional Random Fields: Probabilistic Models for Segmenting and Labeling Sequence Data, in Proceedings of the International Conference on Machine Learning, pp. 282-289 (2001)

[Li 98] Li, H.: A Probabilistic Approach to Lexical Semantic Knowledge Acquisition and Structural Disambiguation, $\mathrm{PhD}$ thesis, The Graduate School of Science of the University of Tokyo (1998)

[Liang 05] Liang, P.: Semi-Supervised Learning for Natural Language, Master's thesis, Massachusets Institute of Technology (2005)

[Manber 91] Manber, U. and Myers, G.: Suffix Arrays: a New Method for On-line String Searches, SIAM Journal on Computing, Vol. 22, pp. 935-948 (1991)

[Marcus 94] Marcus, M., Kim, G., Marcinkiewicz, M. A., MacIntyre, R., Bies, A., Ferguson, M., Katz, K., and Schasberger, B.: The Penn Treebank: Annotating Predicate Argument Structure, Human Language Technology, pp. 114-119 (1994)

[McCallum 00] McCallum, A., Freitag, D., and Pereira, F.: Maximum Entropy Markov Models for Information Extraction and Segmentation, in Proceedings of the International Conference on Machine Learning, pp. 591-598 (2000)

[Mochihashi 09] Mochihashi, D., Yamada, T., and Ueda, N.: Bayesian Unsupervised Word Segmentation with Nested Pitman-Yor Language Modeling, in Proceedings of the Joint Conference of the Annual Meeting of the Association for Computational Linguistics and the International Joint Conference on Natural Language Processing of the Asian Federation of Natural Language Processing, pp. 100-108 (2009)

[Ng 04] Ng, H. T. and Low, J. K.: Chinese Part-of-Speech Tagging: One-at-a-Time or All-at-Once? Word-Based or Character-Based?, in Proceedings of the Conference on Empirical Methods in Natural Language Processing, pp. 277-284 (2004)

[Ratner 87] Ratner, N. B.: The Phonology of Parent - Child Speech, Vol. 6, pp. 159-174, Erlbaum (1987)

[Rissanen 78] Rissanen, J.: Modeling by Shortest Data Description, Aulomatica, Vol. 14, pp. 465-471 (1978)

[Saffran 96] Saffran, J. R., Aslin, R. N., and Newport, E. L.: Statistical Learning in 8-month-old Infants, Science, Vol. 274, pp. 19261928 (1996)

[Tsuboi 08] Tsuboi, Y., Kashima, H., Oda, H., Mori, S., and Matsumoto, Y.: Training Conditional Random Fields Using Incomplete Annotations, in Proceedings of the 22nd International Conference on Computational Linguistics, pp. 897-904 (2008)

[Yu 00] Yu, H.: Unsupervised Word Induction using MDL Criterion, in Proceedings of tne International Symposium of Chinese Spoken Language Processing (2000)

[Zelenko 09] Zelenko, D.: Combining MDL transliteration training with discriminative modeling, in Proceedings of the 2009 Named Entities Workshop: Shared Task on Transliteration, pp. 116-119 (2009)

[Zhikov 10] Zhikov, V., Takamura, H., and Okumura, M.: An Efficient Algorithm for Unsupervised Word Segmentation with Branching Entropy and MDL, in Proceedings of the 2010 Conference on Empirical Methods in Natural Language Processing, pp. 832-842 (2010)

[Zipf 49] Zipf, G. K.: Human Behavior and the Principle of Least Effort, Addison-Wesley (1949)

〔担当委員：小町 守〕

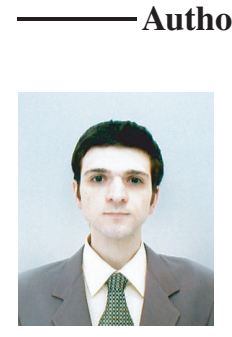

Author's Profile

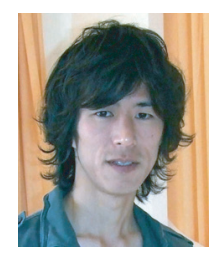

\section{Zhikov, Valentin}

received B.A. in Japanese Studies from Sofia University, Bulgaria in 2006, and M.Eng. in Computational Intelligence and Systems Science from Tokyo Institute of Technology in 2010. Currently employed by Ontotext AD, Bulgaria. Research interests include computational linguistics, semantic annotation and search, natural language interfaces to the semantic web, and general-purpose machine learning.

\section{Takamura, Hiroya (Member)}

received B.E. and M.E. from the University of Tokyo in 1997 and 2000 respectively (in 1999 he was a research student at Technische Universitaet von Wien). He received Dr. Eng. from Nara Institute of Science and Technology in 2003. He was an assistant professor at Tokyo Institute of Technology from 2003 to 2010 . He is currently an associate professor at Tokyo Institute of Technology. His current research inter est is computational linguistics. He is a member of the Information Processing Society of Japan and the Association for Computational Linguistics.

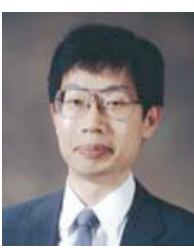

\section{Okumura, Manabu (Member)}

received B.E., M.E. and Dr. Eng. from Tokyo Institute of Technology in 1984, 1986 and 1989 respectively. He was an assistant at the Department of Computer Science, Tokyo Institute of Technology from 1989 to 1992, and an associate professor at the School of Information Science, Japan Advanced Institute of Science and Technology from 1992 to 2000. He was also a visiting associate professor at the Department of Computer Science, University of Toronto from 1997 to 1998 . He is currently a professor at Precision and Intelligence Laboratory, Tokyo Institute of Technology. His current research interests include natural language processing, especially automatic text summarization, computer assisted language learning, sentiment analysis, and text data mining. He is a member of the Information Processing Society of Japan, the Japanese Society for Artificial Intelligence, the American Association for Artificial Intelligence, the Association for Computational Linguistics, and the Japanese Cognitive Science Society.

Received July 25, 2012. 